\title{
Modelling the Stock Price Volatility Using Asymmetry Garch and Ann-Asymmetry Garch Models
}

\author{
Henry Njagi, Anthony Gichuhi Waititu, Anthony Wanjoya \\ Department of Statistics and Actuarial Sciences, Jomo Kenyatta University of Agriculture and Technology, Nairobi, Kenya
}

\section{Email address:}

hnjagim@gmail.com (H. Njagi), agwaititu@gmail.com (A. G. Waititu), awanjoya@gmail.com (A. Wanjoya)

\section{To cite this article:}

Henry Njagi, Anthony Gichuhi Waititu, Anthony Wanjoya. Modelling the Stock Price Volatility Using Asymmetry Garch and AnnAsymmetry Garch Models. International Journal of Data Science and Analysis. Vol. 4, No. 4, 2018, pp. 46-52. doi: $10.11648 / j . i j d s a .20180404 .11$

Received: September 19, 2018; Accepted: October 9, 2018; Published: October 23, 2018

\begin{abstract}
The stock price in developing countries, especially in Kenya, has become one of the market that supports the economy growth of a country. Due to the political instabilities in the Kenyan contest, stock price markets have been affected. As a consequence of the instabilities in the financial markets, this paper model the volatility associated with the stock price for a one day ahead volatility forecast which will help in risk control in the market. This is accomplished by using the asymmetry GARCH and ANN-asymmetry GARCH models. The residuals obtained from artificial neural network are used when fitting ANN- asymmetry GARCH models. It was found that returns on the selected companies in NSE are categorized by volatility clustering, leptokurtosis and asymmetry. In the modelling, we further examine the performance of the leading alternatives with the daily log returns residuals of the leading companies in Kenyan stock market (PAFR, PORT and EGAD) from the period January 2006 to November 2017 for trading days excluding weekends and holidays. The root mean squared error indicated that among the available models i.e. ANN-EGARCH model, GJR-GARCH and EGARCH model, ANN-GJR-GARCH model performed better in modelling and forecasting the stock price volatility in Kenyan contest. The paper demonstrates that combined machine learning and statistical models can effectively model stock price volatility and make reliable forecasts.
\end{abstract}

Keywords: Volatility, Rmse, Ann and Asymmetry Garch Models

\section{Introduction}

Stock price is the highest or the lowest amount that a stock can be bought. The growth of economy draws attention in the field of technology. Tayal et al. stated that big data; stock price being one of the big data becomes the buzzword in the field of technology for some time now [1]. Siegel emphasized that a little prediction goes a long way [2]. Due to the growth in technology, machine learning had been used in prediction of the stock prices. Stock prices in the financial markets; have proved to be very turbulent. There had been a major concern about which methods can be used to measure the price volatility. GARCH (Generalized Autoregressive Conditional Heteroscedasticity) models have been used to measure the volatility and especially asymmetry GARCH models for their capability to model financial data since financial data exhibits heavy tails. Since the problem of identifying the best statistical model in modelling volatility has been an issue, this study shows the capability of combined models and specifically the use of artificial neural network together with asymmetric models in modelling the volatility.

There are basically two broad methods of modelling volatility namely; analytical and historical approach. The analytical approach makes an assumption of distribution which is known for the returns. Actually, since the returns experience heteroskedasticity and serial correlation, the distribution of the returns is modelled. The historical approach majors only on the empirical distribution of the past information.

The asymmetry GARCH models address the challenge of heavy tails in financial data and can be further used to forecast the volatility, the main aim of this work is to comprehend the major strengths and limitations of the GARCH models and Artificial Neural Network in estimating the volatility. Our concern in this work is on the Kenyan Stock Price market. A period spanning from January 2006 to November 2017 was used. Companies used were Eaagads 
Ltd, Pan Africa Insurance Holdings Ltd and E. A. Portland Cement Ltd. Due to the uncertainties that keep on arising due to instability of different factors such as political instability in Kenya, the study saw a need to model the volatility.

The remainder concerns of this paper comprises part 2 explores past works that exist, part 3 explores the GARCH family models and Artificial Neural Network and outlines basic calculation on how to do forecasting of the stock price return volatility based on the asymmetry GARCH models and Artificial-Asymmetry GARCH models. Part 4 outlines the data description used in this work. Part 5 gives the general results. Part 6 provides statistical comparisons of the available models. Lastly, part 7 provides a conclusion and recommendations for further studies.

\section{Literature Review}

Financial time series contain an element of complex dynamic system with a high volatility, this make financial time series different from other time series analysis. There are some statistical regularities that are considered to be consistent empirical observations which are accepted and used in many contexts. There are some patterns that have been identified with financial time series. These patterns are very important for correct model specification, estimation and finally forecast, they include volatility clustering, stock price exhibiting excess kurtosis, i.e. fatter tails than those of normal distribution, stock price movement exhibiting negative correlation with volatility as Knight and Sachell [3]. Stock returns have effect of positive or negative skewness. This effect is used to explain the departure from symmetry of the stock price returns. Due to the developed interest and the attractiveness of modelling stock price volatility, different models have been used especially by the academicians. GARCH-type models have been proposed to incorporate more effect on the field of volatility modelling. It's worth noting that, GARCH models with t-student distribution are able to capture the fat tails in financial data. The challenge with this is that the asymmetric feature of the returns such as leverage effects and volatility persistence are not captured since the symmetry models are able to capture leptokurtosis and volatility clustering.

The problem with symmetry GARCH was solved by Nelson by frosting the Exponential GARCH [4]. Nelson claimed that the variance of stock price returns responded differently to both positive and negative returns. The claim was found to be true and conclude that there was a negative relationship between excess returns and the variance of stock markets. Glosten, Jagannathan and Runkel introduced GARCH (GJR-GARCH) model which is a type of asymmetry GARCH [5]. Brooks stated that GJR model is an extension of GARCH that allows a different reactions for the negative unexpected returns and positive unexpected returns to the stock price volatility [6].

Financial stock market reacts more to "bad news" than "good news". This condition contributes to one of the financial time series pattern; the leverage. Nelson stated the effect of bad and good news on volatility and found out that there was an asymmetry in the volatility of the stock price markets [4]. Their study outlined that bad news create more volatility as compared to the good news.

Lesaona, Makhwiting and Sigauke modeled volatility and financial market risk of Johannesburg stock exchange (JSE) returns [7]. The process included two steps. The first step was estimation of $\operatorname{ARMA}(0,1)$ model for the mean returns, second step was to fit different univariate GARCH-type models for conditional volatility; $\operatorname{GARCH}(1,1)$, T$\operatorname{GARCH}(1,1)$, EGARCH$(1,1)$ and GARCH-M(1,1) models. The results showed the presence of $\mathrm{ARCH}$ and leverage effects in JSE returns over the specified sample period. The evaluation on the forecast indicated that $\operatorname{ARMA}(0,1)$ GARCH $(1,1)$ performed best in forecasting out-of-sample returns.

Alagidede examined the behavior of stock returns in six African markets (Kenya, Egypt, Morocco, Tunisia, Nigeria, South Africa and Zimbabwe) [8]. They found that financial time series features i.e. volatility clustering, asymmetry and leptokurtosis are present in African markets stock index returns. Emenik K.O. and Aleke Studied the return volatility of stock returns of the Nigerian Stock Exchange using GARCH $(1,1)$, EGARCH $(1,1)$ and GJR-GARCH $(1,1)$ over the period January $2^{\text {nd }}$, the year 1996 to $30^{\text {th }}$ of December 2011[9]. They found evidence of long memory and volatility clustering in the returns. However, the GJRGARCH and E-GARCH produced conflicting results about whether there is asymmetry in the returns.

Generally research has found that GARCH-type models are able to model the features observed in return behavior. Brooks highlighted that GARCH models are able to account for the mean-reverting tendency found in volatility series [6]. The study observed that if volatility series are low relative to their historic average, they will have a tendency to rise back towards the average, and this phenomenon is modelled by GARCH.

Forecasting accuracy of the random walk model, historical mean model, moving average model, exponential smoothing model, exponentially weighted moving average model, a simple regression model, two standard GARCH models and two asymmetric GJR-GARCH models in forecasting volatility of the Australia stock market [10]. In the study, it was found that the superiority of a model's forecasting capabilities is strongly influenced by the choice of error statistic used to assess the accuracy of the model. Thus, they conclude no single model is best but found the GJR-GARCH model slightly superior to the simpler models for predicting Australian monthly stock index volatility.

The volatility on the Ghana Stock Exchange was carried on using the random walk, GARCH $(1,1)$, EGARCH $(1,1)$ and TGARCH $(1,1)[11]$. The study rejects the random walk hypothesis for GSE returns and finds that the returns are categorized by the main stylized features found in financial data, i.e. leptokurtosis, volatility clustering and asymmetric effects. The GARCH type models had been used widely especially with Bangladesh Bank which is very sensible to 
the stock market, several researchers developed hypothesis and analysis. One of the notable stock market in Bangladesh is Dhaka Stock Exchange (DSE) and most of the work that focused on market volatility and their returns [12].

Regarding the critical issue of volatility of stock price returns, a good number of researchers have become involved in modeling and making comparisons of which model is good in forecasting the stock price volatility. A number of papers have examined thoroughly. Poon and Grager have evidently mentioned that GARCH performs better and dominates the ARCH models [13]. Dutta found out that asymmetry GARCH models estimate better than symmetry models [14]. Liu, et al. did a good work and investigated that the EGARCH model achieved superior performance in predicting stock market volatility [15]. Onwukwe, et al. mentioned that the GJR-GARCH model significantly outperforms the existing models. This was done by testing the results of error statistics and model accuracy methods [16].

Over a long period, machine learning techniques have been used for financial time series analysis and predictions. One of the most complex machine learning employed is Artificial Neural Networks (ANN) [17]. ANN have been used as the most popular machine learning techniques since early 1990's. The standard statistical models have been used in the field of financial time series analysis and prediction, but artificial neural network have gained popularity. Schoeneburg made an investigation about the possibility of price prediction by different algorithms of neural [18]. The results indicated that artificial neural network model can be successfully applied in making the predictions of data in the field of financial time series. Combined models, artificial neural network were combined with asymmetric model for a better performance in stock price returns volatility.

\section{Methodology}

\subsection{Volatility Definition}

Volatility is the amount of uncertainty, risk/fluctuation about the size of changes in a security's value over a period of time.

\subsubsection{Standard Garch-Type Model}

The GARCH (p, q) model is generally given by [19]:

$$
r_{t}=\mu+a_{t}, a_{t}=\sigma_{t} \varepsilon_{t}
$$

where $\delta_{\mathrm{t}}$ is given by;

$$
\sigma_{\mathrm{t}}^{2}=\alpha_{0}+\sum_{\mathrm{i}=1}^{\mathrm{p}} \alpha_{\mathrm{i}} \varepsilon_{\mathrm{t}-\mathrm{i}}^{2}+\sum_{\mathrm{i}=1}^{\mathrm{q}} \beta_{\mathrm{i}} \delta_{\mathrm{t}-\mathrm{j}}^{2}
$$

Where $r_{t}$ the logarithmic returns, $\mu$ are the return's mean value, $a_{t}$ are the mean-equation innovations. In order to ensure stationary of loss series, $\alpha_{1}+\beta_{1}<1$. The GARCH (1, 1) model is the most commonly used in financial data. The form of $\operatorname{GARCH}(1,1)$ model is given by:

$$
\sigma_{\mathrm{t}}^{2}=\alpha_{0}+\alpha_{1} \varepsilon_{\mathrm{t}-1}^{2}+\beta_{1} \delta_{\mathrm{t}-1}^{2}
$$

In order to have a positive variances every time, the following restrictions are imposed $\alpha_{0}>0$, and $\alpha_{1}, \beta_{1} \geq 0$.

The standard GARCH model captures volatility clusters and heavy tails. Standard GARCH distributions is symmetric and therefore they fail to model the leverage effect. To address this problem, many asymmetry GARCH models have been proposed such as E-GARCH model [4]. The GJRGARCH model, an asymmetry model that was proposed by Glosten Jagannathan and Runkle [5].

\subsubsection{Asymmetric Garch Models}

Glosten Jagannathan and Runkle noted that the prices of financial assets often react more to bad news than good news and such a condition leads to leverage effect [5]. To get the asymmetry property of volatility, asymmetry models was developed and are called asymmetry GARCH models. In this paper, the following asymmetric GARCH Models will be used to calculate the volatility; Exponential GARCH and the Glosten Jagannathan and Runkle GARCH model.

\subsubsection{Exponential Garch Model (Egarch)}

The EGARCH model for capturing the asymmetry nature of returns was developed to solve three main weaknesses of the symmetric GARCH model:

Non sensitivity to asymmetric response of volatility to shocks

Parameter restrictions that ensures conditional variance positivity

Difficulty in measuring the persistence in stationary time series

The form of EGARCH (p, q) is given by:

$$
\ln \delta_{t}^{2}=\omega+\alpha_{1}\left|\frac{x_{t-i}}{\sigma_{t-i}}\right|+\gamma_{i} \frac{x_{t-i}}{\sigma_{t-i}}+\sum_{i=1}^{q} \beta_{j} \ln \sigma_{t-j}^{2}
$$

Where $\gamma$ is the asymmetric response parameter.

In this paper we will use the simplest form of EGARCH which is the EGARCH $(1,1)$ model which is given by:

$$
\ln \delta_{t}^{2}=\omega+\alpha_{1}\left|\frac{x_{t-1}}{\sigma_{t-1}}\right|+\gamma_{i} \frac{x_{t-1}}{\sigma_{t-1}}+\beta_{1} \ln \sigma_{t-1}^{2}
$$

\subsubsection{GJR-Garch Model (Glosten Jagannathan and Runkle Garch)}

The GJR-GARCH model was proposed by Glosten Jagannathan and Runkle. The model takes into consideration that leverage effect based on the state of past innovation. Volatility equation of GJR-GARCH (p, q) is given by:

$$
\sigma_{t}^{2}=\omega+\sum_{i=1}^{p} \alpha_{i} \frac{x_{t-i}}{\sigma_{t-i}}+\sum_{j=1}^{q} \beta_{j} \sigma_{t-j}^{2}+\gamma_{i} I_{t-i} \frac{x_{t-i}}{\sigma_{t-i}}
$$

Where $\alpha, \beta$ and $\gamma$ are constants, and $\mathrm{I}$ is a dummy variables. The parameters of the model are assumed to be non-negative and that $\frac{\alpha+\gamma+\gamma}{2}<1$.

\subsubsection{Ann - Gjr - Garch Model}

Artificial neural network is a collection of transfer functions that involves two types of variables i.e. output variables and input variables, the relationship of the output variables to the input variables is explained by artificial 
neural network [18].

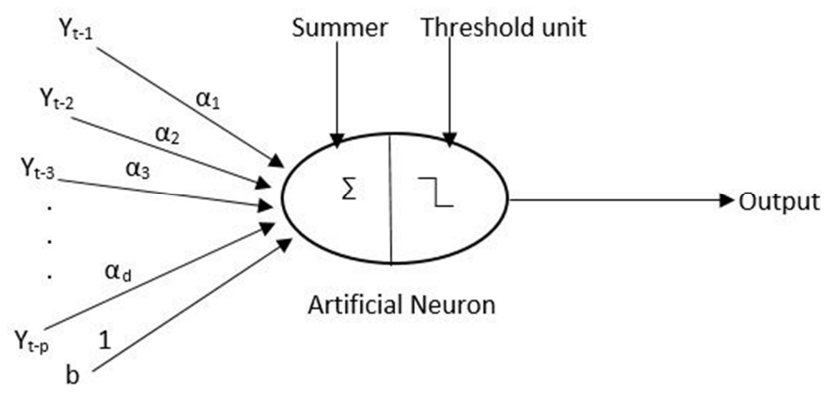

Figure 1. Single neuron with p inputs.

Where;

$$
\text { Summer }=\sum_{i=1}^{p} \alpha_{i} y_{t-i}
$$

And $\alpha_{1}, \alpha_{2}, \alpha_{3}, \ldots, \alpha_{d}$ are the weights of connections, $y_{t-1}, y_{t-2}, y_{t-3}, \ldots, y_{t-p}$ are the inputs and $\mathrm{b}$ is the bias.

The following is a mathematical representation showing how the output and input are related;

$$
y_{t}=w_{0}+\sum_{j=1}^{q} \beta_{j} g\left(w_{0 j}+\sum_{i=1}^{p} \alpha_{i j} y_{t-i}\right)+\varepsilon_{t}
$$

where $\beta_{\mathrm{j}}(\mathrm{j}=1,2,3 \ldots, \mathrm{q})$ are the weights which can be learned from the labelled training dataset [20]. The model parameters, $\alpha_{i j}(i=1,2,3, \ldots, p ; j=1,2,3, \ldots, q), p$ is the total number of nodes in the input layer; $\mathrm{q}$ is the number of nodes in the hidden layer. $\varepsilon_{t}{ }^{\prime} s$ are the residuals. The connections between nodes of the hidden layer is determined by the function $\mathrm{g}($.). This function is used as the hidden unit activation function to enhance the nonlinearity of the model. The applied transfer function in this paper is logistic function which is given as

$$
g(x)=\operatorname{Sig}(x) \frac{1}{1+\exp (-x)^{\prime}}
$$

The researchers in the field of artificial neural network found out that, provided there is sufficient nodes in the first hidden layer of the ANN, higher layers are unnecessary to establish a satisfactory connection between the raw-data inputs and the final output. In this paper, single hidden layer ANN is employed where there is an optimal selection of the number of the nodes. The optimum ANN architecture employed was 8-2-1. That is 8-nodes in the input layer, 2nodes in the hidden layer and 1-node in the output layer.

$$
\begin{array}{r}
\sigma_{t}^{2}=\omega+\sum_{i=1}^{p} \alpha_{i} \frac{x_{t-i}}{\sigma_{t-i}}+\sum_{j=1}^{q} \beta_{j} \sigma_{t-j}^{2}+\gamma_{i} I_{t-i} \frac{x_{t-i}}{\sigma_{t-i}}+ \\
\sum_{k=1} \xi_{k} \varphi\left(z_{t} \tau_{k}\right)
\end{array}
$$

Where;

$$
\begin{gathered}
\varphi\left(z_{t} \tau_{k}\right)=\left[1+\exp \left(\tau_{k .0 .0}+\sum_{d=1}^{n}\left[\sum_{w=1}^{m}\left(\tau_{k . d . w_{t-d}} z_{t-d}^{w}\right)\right]^{-1}\right.\right. \\
z_{t-d}=\left[\varepsilon_{t-d}-E(\varepsilon)\right] / \sqrt{E\left(\varepsilon^{2}\right)} \\
\frac{1}{2} \tau_{k . d . w} \sim \text { uni }[-1,+1]
\end{gathered}
$$

In this section, the estimation of the parameters follows the procedure implemented in this section.

The two steps that were implemented in this paper are summarized as:

Fit the stock price returns to artificial neural network model and extract its residuals.

Fit asymmetry GARCH models to the obtained residuals from artificial neural network in order to obtain the parameters.

\subsection{Conditional Distributions}

Since returns are not normal in distribution, the occurrence of the so-called fat-tails distributions warrants the use of nonnormal distributions such as the student t-distribution, generalized error distribution and skewed student $t$ distribution. In this paper, student $\mathrm{t}$-distribution was implemented in the asymmetry GARCH models.

\subsubsection{Student-t Distribution}

Bollerslev introduced the student $t$ distribution [19]. The form is given in equation (14).

$$
f\left(z_{t}, t\right)=\frac{\Gamma\left(\frac{u+1}{2}\right)}{\Gamma\left(\frac{u}{2}\right) \sqrt{\pi(u-2)}}\left(1+\frac{z_{t}^{2}}{u-2}\right)^{-\frac{u+1}{2}}
$$

Where the function $\Gamma($.$) is gamma. Since student \mathrm{t}$ distribution is not the sole distribution that accounts for fat tails in financial data, the generalized error distribution also does account for fat tails

\subsubsection{Estimation Under the Asymmetry Garch}

Here we discuss the estimation of parameters and the forecasting using asymmetry GARCH models discussed in section 3.1.2.

The forecast for one-day ahead variance for the EGARCH model is given by equation (15)

$$
\ln \delta_{t+1}^{2}=\omega+\alpha_{i}\left|\frac{x_{t-i+1}}{\sigma_{t-i+1}}\right|+\gamma_{i} \frac{x_{t-i+1}}{\sigma_{t-i+1}}+\beta_{j} \ln \sigma_{t-j+1}^{2}
$$

The forecast for one-day ahead variance under ANN-GJRGARCH model is given by equation (16)

$\sigma_{t+1}^{2}=\omega+\sum_{i=1}^{p} \alpha_{i} y_{t-i+1}+\sum_{j=1}^{q} \beta_{j} y_{t-j+1}+\gamma_{i} I_{t-i+1} y_{t-i+1}$

Therefore to compute the one-step ahead stock price volatility forecast under the assumed distribution was accomplished by equation (15) and (16).

\section{Data Description}

To compare the robustness of the asymmetry-GARCH and ANN-asymmetry GARCH models we choose the context of Nairobi Stock Exchange Market as the base of analysis. The data employed was the 2782 observations of the daily stock prices of the Kenyan Market covering the period from $2^{\text {nd }}$ of January 2006 to $13^{\text {th }}$ of November 2017

The data excluded weekends and public holidays. The source of the data was the NSE website. The daily stock prices were transformed into daily log returns by applying: 


$$
\mathrm{R}_{\mathrm{t}}=\log \left(\frac{\mathrm{P}_{\mathrm{t}}}{\mathrm{P}_{\mathrm{t}-1}}\right)
$$

Where $\mathrm{P}_{\mathrm{t}}$ is the stock price at time $\mathrm{t}$ and $\mathrm{P}_{\mathrm{t}-1}$ the stock price at time $t-1$.

\section{Empirical Result}

Figure 2 shows the stock prices for the Kenyan stock market. As depicted in the figure, a decline is observed in 2007. A sharp decline is observed in 2008 for all companies because of the post-election violence that occurred then. Consistent instability is seen from 2008 to 2010 which is attributed to the violence that occurred after the elections in 2007. Stability in the prices is observed from 2016 to July 2017 just before the general elections were held. The daily logarithm returns in Figure 2 for each company, indicates periods of very high volatility and mostly volatility clustering. This illustrates the high volatility of developing country financial market.

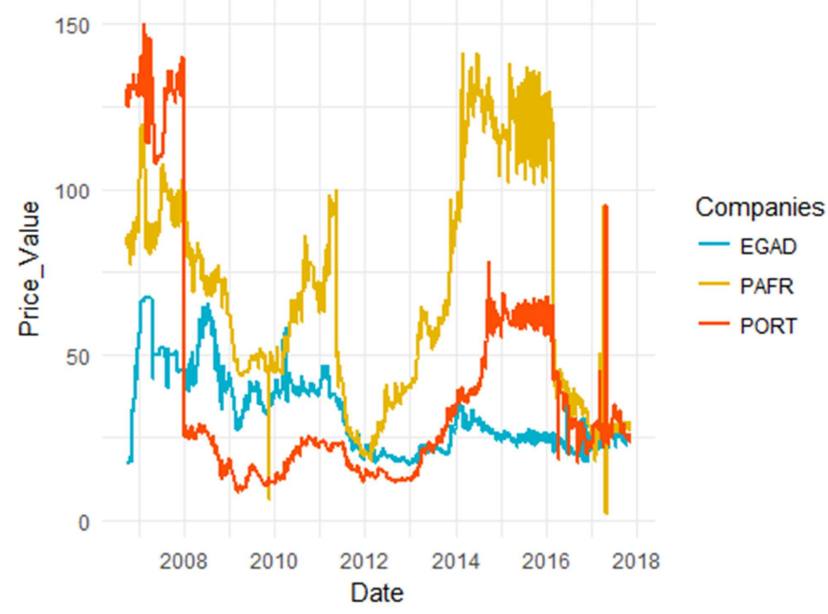

Figure 2. Time series plots for the daily stock prices.

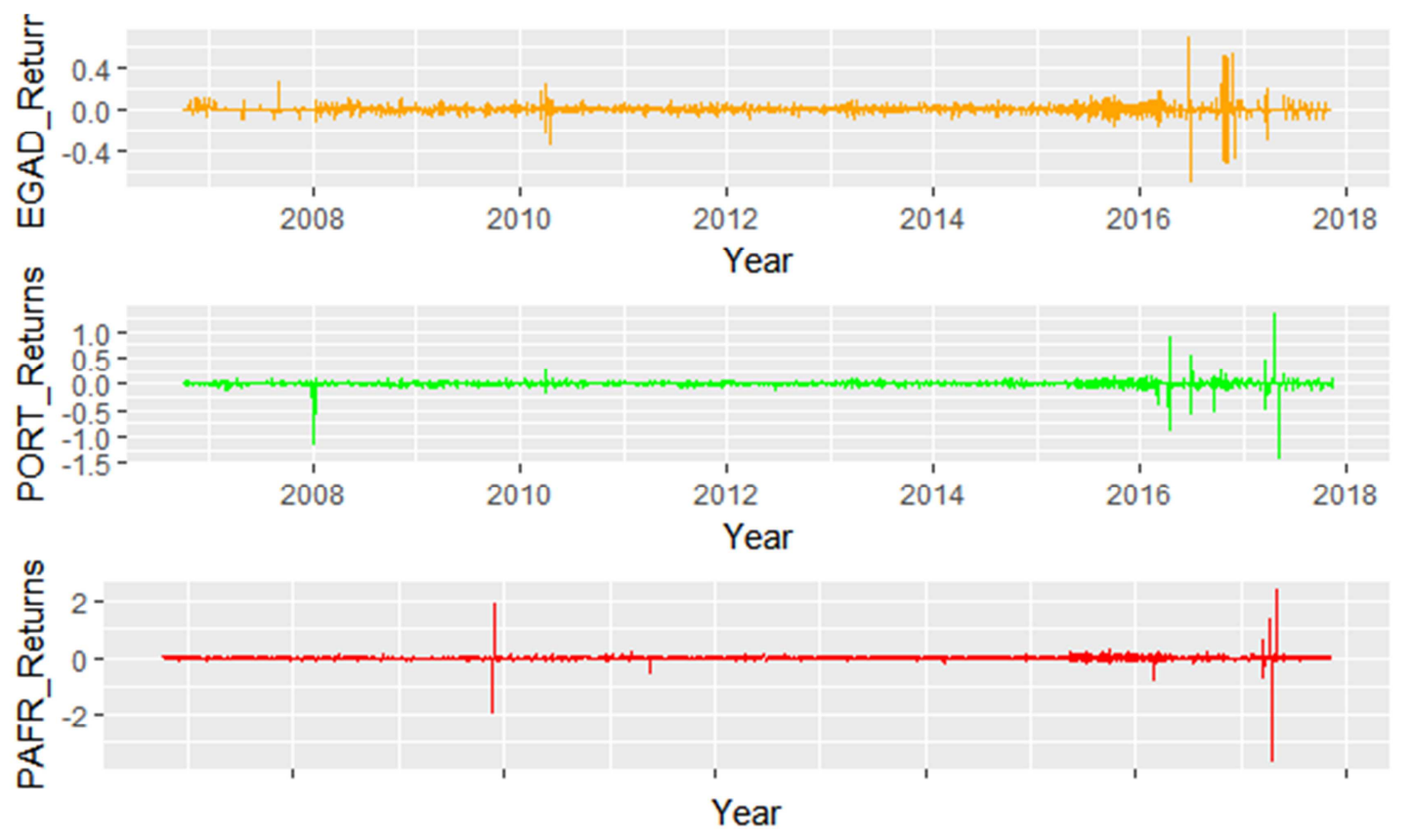

Figure 3. Time series plot for the daily returns for exchange rates.

Figure 3 indicates the log returns of the stock prices.

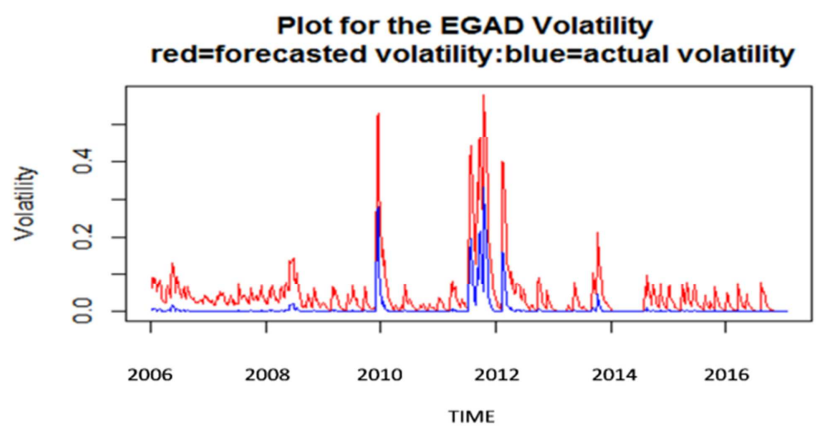

Figure 4. Plot for the actual versus forecasted values for PORT data.
Plot for the PORT Volatility red=forecasted volatility:blue=actual volatility

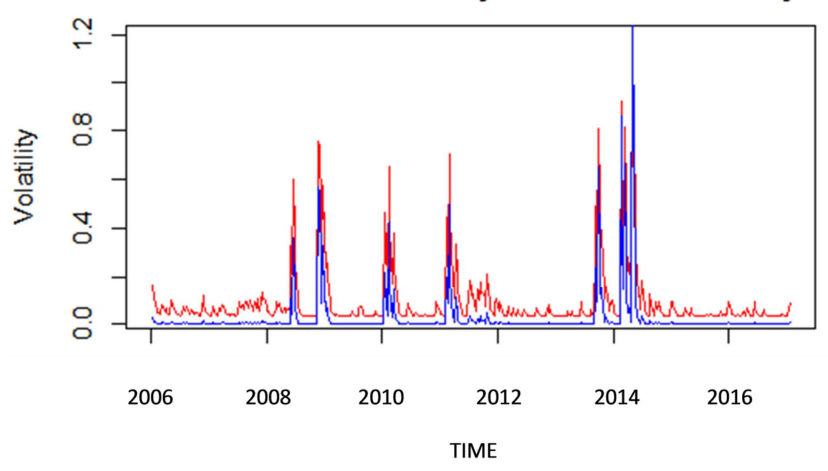

Figure 5. Plot for the actual versus forecasted values for PORT data. 
Table 1. Summary Statistics for Stock Price Returns.

\begin{tabular}{llll}
\hline Statistic & EGAD & PORT & PAFR \\
\hline Size & 2782 & 2782 & 2782 \\
Mean & 0.0001 & -0.0006 & -0.0004 \\
Minimum & -0.69 & -1.40 & -3.68 \\
Maximum & 0.68 & 1.33 & 2.39 \\
Std. Deviation & 0.05 & 0.06 & 0.11 \\
Skewness & -0.22 & -2.97 & -8.86 \\
Kurtosis & 75.34 & 239.85 & 577.79 \\
Jarque-Bera & 658710 & 6679700 & 38777000 \\
JB p-value & $<0.0002$ & $<0.0002$ & $<0.0002$ \\
\hline
\end{tabular}

Table 1 shows the summary statistics of the daily returns of the stock price. An upward movement of the stock price market is depicted by the negative loss. In addition it is found out that the three companies experience more frequent negative shock. The high excess kurtosis observed across all companies confirm that fat tails in return distribution. The J$\mathrm{B}$ test for normality of the returns was performed. A p-value less than 0.05 gives a strong evidence of the returns being non-normal.

Table 2 presents optimal variance equation of the stock price returns. The optimal variance equation were chosen based on the minimum BIC value. Generally, the models portray a similar picture of volatility over time. The optimal GARCH model for the stock price is the ANN-GJR-GARCH model with student $t$ distribution. This model performed the best for the data of the selected companies

Table 2. Model specifications employed.

\begin{tabular}{lllll}
\hline Company name & EGARCH $(\mathbf{p}, \mathbf{q})$ & GJR-GARCH $(\mathbf{p}, \mathbf{q})$ & ANN-EGARCH $(\mathbf{p}, \mathbf{q})$ & ANN-GJR-GARCH $(\mathbf{p}, \mathbf{q})$ \\
\hline EGAD & 1,1 & 1,1 & 1,1 & 1,1 \\
PORT & 1,1 & 1,1 & 1,1 & 1,1 \\
PAFR & 1,1 & 1,1 & 1,1 & 1,1 \\
\hline
\end{tabular}

From table 3, the train MSE is obtained by getting the mean squared error between the fitted values and the actual values. The test MSE is obtained by getting the MSE between the forecast and the test data. The mean squared error both for training and testing is acceptable.

Table 3. Table of mean squared error of 8-2-1.

\begin{tabular}{llll}
\hline EGAD COMPANY & & & \\
\hline No of inputs & 8 & Total train MSE & 0.0094 \\
No of layers & 1 & Total test MSE & 0.0152 \\
No of neurons & 2 & & \\
No of outputs & 1 & & \\
PORT COMPANY & & & 0.0052 \\
No of inputs & 8 & Total train MSE & 0.0091 \\
No of layers & 1 & Total test MSE & \\
No of neurons & 2 & & \\
No of outputs & 1 & & 0.0002 \\
PAFR COMPANY & & & 0.0089 \\
No of inputs & 8 & Total train MSE & \\
No of layers & 1 & Total test MSE & \\
No of neurons & 2 & & \\
\hline No of outputs & 1 & & \\
\hline
\end{tabular}

The volatility plots were done to find out whether there is a systematic pattern between the actual volatility and the forecasted volatility. The plots shows that the model captures the volatility well [19].

\section{Comparing the Forecast}

The comparison of different models in forecasting were used. The machine learning and statistical models in forecasting were evaluated by different researchers and found that machine based models performs better. Sigo et al. found that the forecasting accuracy was higher in the case of k-nn algorithm model than that of logistic regression method [21].

The model forecasting aptitude was evaluated using the squared error in the root mean. Root Mean Squared Error is the most favoured statistics measure among the Researchers, practitioners and has even stronger preference among the academicians:

$$
\operatorname{RMSE}=\sqrt{\frac{\sum_{\mathrm{t}=1}^{\mathrm{T}}\left(\widehat{y}_{\mathrm{t}}-\mathrm{y}_{\mathrm{t}}\right)^{2}}{\mathrm{~T}}}
$$

Where $y_{t}$ is the actual value and $\hat{y}_{t}$ is the predicted value. The advantage of using the Root Mean Squared Error is that it gives the errors equal weights, irrespective of any time period.

Table 4. Table of root mean squared error.

\begin{tabular}{lllll}
\hline Model & EGAD & PORT & PAFR & Rank \\
\hline EGARCH(1,1) & 0.0482 & 0.0624 & 0.1111 & 4 \\
ANN-EGARCH $(1,1)$ & 0.0531 & 0.0411 & 0.0420 & 2 \\
GJR-GARCH $(1,1)$ & 0.0623 & 0.0480 & 0.1104 & 3 \\
ANN-GJR-GARCH $(1,1)$ & 0.0145 & 0.0314 & 0.0484 & 1 \\
\hline
\end{tabular}

Table 4 shows the Root Mean Squared Error (RMSE). The model that gives the smallest RMSE is the best model among the list of the models. The root mean squared errors indicated in table 4 shows that ANN-GJR-GARCH model was the most appropriate model in forecasting the stock price return volatility for the data available for the three companies as it recorded the smallest RMSE.

\section{Conclusions}

The aim of this paper was to evaluate which model performs better in forecasting the stock price volatility using asymmetry GARCH and ANN asymmetry GARCH forecast models. The findings of the study are that the ANN-GJRGARCH model with t-distribution is the best performing model in estimating and forecasting the volatility for the EGAD data, for the PORT data and PAFR data in Kenyan market. Further research should include the factors that affect the stock price volatility. 


\section{References}

[1] S. N. N. \&. S. K. Tayal, "Role of big data in make in India.," Advances in Intelligent Systems and Computing, pp. 564, 531437, 2018.

[2] E. Siegel, "Predictive analytics: The power to predict who will click, buy, lie or die," Wiley India Pvt. ltd., New Delphi., pp. ISBN: 978-81-265-5977-0, 2016.

[3] F. BLACK, "Studies of stock price volatility changes," Proceedings of the 1976 meetings of the American Statistical Association. Business and Economical Statistical section., pp. 177-181, 1976.

[4] D. B. Nelson, "Conditional Heteroscedasticity in Asset Returns: A New Approach," Econometrica, vol. 59, no. 2, pp. 347-370, 1991.

[5] Glosten, Jagannathan and Runkel, "The Relationship between the expected value and volatility of nominal excess returns," Journal of Finance, vol. 48, pp. 1779-1801, 1993.

[6] C. BROOKS, "Introductory Econometrics for Finance (2e).," Cambridge University Press:, 2008.

[7] M. M. M. R. a. S. C. LESAONA, "Modelling volatility and financial market risk of shares on the Johannesburg stock exchange.," African Journal of Business Management., pp. 6: 8065-8070., 2012.

[8] P. Alagidede, "Return behaviour in Africa's emerging equity markets.," Quarterly Review of Economics and Finance, pp. 51(2): 133-140, 2011.

[9] K. a. A. S. Emenik, "Modeling asymmetric volatility in the Negerian Stock Exchange.," European Journal of Business and Management, pp. 4(12): 52-61, 2012.

[10] T. a. F. R. Brailsford, "An evaluation of volatility forecasting techniques.," Journal of Banking and Finance, pp. 20:419-438, 1996.

[11] F. a. F. O. Magnus, "Modelling and Forecasting Volatility of
Returns on the Ghana Stock Exchange Using GARCH Models.," American Journal of Applied Sciences., pp. 3(10): 2042-2048, 2006

[12] B. N. N. Siddikee M N, "Volatility of Dhaka stock exchange returns by GARCH models.," Asian Business Review,, pp. 8(5): 220-229, 2016.

[13] G. C. W. Poon S H, "Forecasting Volatility in Financial markets:," A review journal of Economic Literature,, pp. 41(2):478-539, 2003.

[14] D. A., "A study of the NSE's volatility for very small period using asymmetric GARCH models. Vilakshan:", The XIMB Journal of Management, pp. 7(2): 107-120, 2010, .

[15] H. J. C. Liu H C, "Forecasting S \& P-100 stock index volatility: The role of volatility asymmetry and distributional assumption in GARCH models.," Expert Systems with Applications, pp. 37(7);4928-4934, 2010,.

[16] B. B. E. E. I. I. O. Onwukwe C E, "On modelling the volatility of Nigerian stock returns using GARCH models.," Journal of Mathematics Research,, pp. 3(4):31-43, 2011,

[17] H. White, "Economic prediction using neural networks: The case of IBM stock prices.," Proceedings of the Second Annual IEEE Conference on Neural Networks, pp. Networks, pp. II: 451 458. New York: IEEE Press, 1988.

[18] E. Schoeneburg, "Stock Price Prediction Using Neural Networks:" A Project Report, Neurocomputing,, pp. Vol. 2, pp. 17-27, 1990.

[19] Bollerslev, "Generalized Autoregressive Condtional Heteroscedasticity," Journal of Econometrics, 1986.

[20] T. L. B. F. Z. W. T. Q. Z. Guo, "Learning sentimental weights of mixedgram terms for classification and visualization.," PRICAI 2016, LNAI 9810, pp. pp. 116-124, 2016.

[21] M. S. M. L. K. \&. V. V. Sigo, "Forecasting the Stock Index Movements of India: Application of Neural Network.," International journal of Soft Computing, pp. 12(2), 120-131, 2017. 